\title{
An Examination of Parameters Affecting Large Eddy Simulations of Flow Past a Square Cylinder
}

\author{
M. R. Mankbadi" and N. J. Georgiadis ${ }^{*}$ \\ Inlet and Nozzle Branch, NASA Glenn Research Center, \\ 21000 Brookpark Rd, Cleveland, $\mathrm{OH} 44135$
}

\begin{abstract}
Separated flow over a bluff body is analyzed via large eddy simulations. The turbulent flow around a square cylinder features a variety of complex flow phenomena such as highly unsteady vortical structures, reverse flow in the near wall region, and wake turbulence. The formation of spanwise vortices is often times artificially suppressed in computations by either insufficient depth or a coarse spanwise resolution. As the resolution is refined and the domain extended, the artificial turbulent energy exchange between spanwise and streamwise turbulence is eliminated within the wake region. A parametric study is performed highlighting the effects of spanwise vortices where the spanwise computational domain's resolution and depth are varied. For $R e=22,000$, the mean and turbulent statistics computed from the numerical large eddy simulations (NLES) are in good agreement with experimental data. Von-Karman shedding is observed in the wake of the cylinder. Mesh independence is illustrated by comparing a mesh resolution of 2 million to 16 million. Sensitivities to time stepping were minimized and sampling frequency sensitivities were nonpresent. While increasing the spanwise depth and resolution can be costly, this practice was found to be necessary to eliminating the artificial turbulent energy exchange.
\end{abstract}

$\begin{array}{cll}\begin{array}{c}\text { Nomenclature } \\ \rho\end{array} & =\text { density } \\ \frac{u}{p} & =\text { velocity vector } \\ E & =\text { pressure } \\ \mu & =\text { dotal energy per unit mass } \\ H & =\text { total enthalpy } \\ T & =\text { temperature } \\ \frac{\tau}{C_{v}} & =\text { shear stress tensor } \\ C_{p} & =\text { specific heat at constant volume } \\ x, y, z & =\text { cartesian coordinates } \\ \Delta x, \Delta y, \Delta z & =\text { grid spacing } \\ U_{r e f} & =\text { reference velocity }\end{array}$

$\begin{array}{cll}\bar{U} & = & \text { mean streamwise velocity } \\ \omega_{x} & = & \text { longitudinal vorticity } \\ u_{r m s}=\sqrt{\overline{u^{\prime} u^{\prime}}} & = & \text { rms streamwise velocity } \\ v_{r m s}=\sqrt{\overline{v^{\prime} v^{\prime}}} & = & \text { rms transverse velocity } \\ w_{r m s}=\sqrt{\overline{w^{\prime} w^{\prime}}} & = & \text { rms spanwise velocity } \\ \frac{u^{\prime} u^{\prime}}{u^{\prime} v^{\prime}} & = & \text { Reynolds normal stress } \\ \bar{C}_{d} & = & \text { Reynolds shear stress } \\ C_{d_{r m s}} & = & \text { mean drag coefficient } \\ C_{l r m s} & = & \text { rms dift coefficient } \\ l_{R} & = & \text { reattachment length } \\ R e & = & \text { Reynolds number } \\ S t & = & \text { Strouhal number }\end{array}$

\section{Introduction}

The close inspection of flow over bluff bodies has been a constant theme throughout the history of fluid mechanics. It will continue to be a benchmark problem in the years to come for all the advances in both the numerical and the experimental subfields of fluids. In part, the reason for this is due to the rich flow physics encapsulated within a simple geometry. The flow phenomena include stagnation, reversal, separation, unsteady interactions within the boundary layer, wake flow, and turbulence. These fundamental flow physics are almost universal, that is they appear in several other more complicated geometries such as flow over a wing, flow in a compressor, rotor-stator interactions of a turbine, mixing of a compressible shear layer with shocks, and jet flows. A good understanding of flow over a bluff body certainly aids the understanding of flow over more complicated configurations. 
In the work herein, the focus is on understanding the flow over a bluff body with emphasis on the numerical uncertainties that arise from truncation of the spanwise extent and spatial resolution of spanwise periodic flows. Although there has been an increase in use of large eddy simulations (LES) in the past decade, the spanwise dimension is often underresolved. Consequently, this may result in imposing a spanwise mode that is nonphysical, and a transfer of energy from the unresolved spanwise turbulence to the resolved transverse and streamwise turbulence. For a square cylinder at Re of 22000, the findings of an LES Workshop are summarized in (Rodi 2006). It notes that a spanwise depth of $2 \mathrm{D}$ is too short to allow for the evolution of three-dimensional structures. Following along that works recommendation of increasing the domain extent from 2D to $4 \mathrm{D}$, the work herein extends the domain to 20D. This work is designed to inform the investigations of geometry-intensive LES in which parametric studies are expensive and often done with Reynolds-averaged Navier Stokes (RANS).

The differences between unsteady RANS (URANS) and high fidelity LES have been highlighted by Rodi (1997) and Hah et al. (2012). LES better predicts the location of separation and captures the unsteady behavior. Although URANS in some cases can accurately capture the time-mean flow, the research literature finds that it fails to accurately capture rms values thereby misrepresenting turbulent flow characteristics. The big difference is URANS inherently uses a large turbulent viscosity to replace all turbulent stresses while LES is using a comparatively much smaller viscosity to only model the subgrid stresses. In effect, then, the only unsteady motion URANS should capture is not turbulence.

Within the LES framework, there are different numerical schemes that can be of the same spatial order as URANS or higher order. Also the use of subgrid scale (SGS) models is more helpful for higher-order methods than for inherently dissipative second order methods (see Eastwood et al., 2009). For some wall bounded flow problems, e.g. a compressible shear layer, the addition of a RANS layer in the near wall region alleviates the Reynolds number constraints on LES to resolve the boundary layer (Georgiadis et al., 2003). There are different ways to blend the two approaches within the LES-RANS approach. Some works do not convert the RANS obtained Reynolds stresses to a time varying function. In those cases, the LES region is simply given a mean profile with no incoming turbulence. Synthetic eddy models have been proposed as a way of converting the Reynolds stresses to a time-varying function with turbulence.

With the recent advancement of computing power, 'practical' LES is becoming more feasible while 'rigorous' LES is still advancing (DeBonis 2007). 'Rigorous' LES tends towards resolving the flow around canonical problems and identifying the physical mechanisms of the general flow field, while 'practical' LES resolves the flow around industrial problems which have a more complex geometry. Consequently, the less CPU intensive sub-class of spectral-like methods is constrained to simple geometries. High order methods that minimize dissipation and dispersion errors are well suited for Rigorous LES (DeBonis 2007), but typically due to the large stencil size and stricter numerical stability criteria are ill suited for complex geometries. The goal of practical LES is to tackle flow physics present in complex industrial problems that cannot be captured accurately by RANS alone. The work herein demonstrates the proficiency of an 'industrial' code at solving a canonical problem such as the square cylinder. The focus of the shear layer problem is on the separated flow, wake, and mixing and less on the attached flow by the walls, whereas the walls around the cylinder herein are separated. Hence the addition of a RANS layer is not advantageous for the square cylinder as was for the compressible shear layer.

LES may be split into two categories: the conventional one utilizes explicit sub-grid scale (SGS) models for the unresolved scales. Alternatively, Eastwood et al. (2009) have demonstrated that for inherently dissipative schemes it is difficult to separate the effects of SGS from the schemes' dissipation. It was also demonstrated that SGS modeling may not be required for second order schemes but are frequently more important for higher order schemes. In relation to SGS modeling, the effects of inflow boundary conditions, problem definition and transition were found to be more influential (Eastwood et al., 2009). Computations which do not use SGS modeling were termed numerical large eddy simulation (NLES) by Pope (2000). The term "implicit LES" (ILES) is also frequently used and was introduced by Boris et al. (1992).

In section II the formulation being evaluated numerically is described. Section III discusses the numerical model, boundary conditions, and grid. The results and discussion in section IV are split into two parts: the first addresses numerical sensitivities to sampling frequency, time step, and mesh. The second part discusses results of a parametric study of the effects of spanwise domain extent and grid isotropy. The conclusions in section V summarize the findings of this work.

\section{Formulation}

The spatially filtered Navier-Stokes equations are solved numerically. The compressible form of the equations is solved in the method employed herein, although it is noted that the flow problem under consideration is incompressible. The viscosity $\mu$ and thermal conductivity $k$, are allowed to vary with temperature. However, for the 
benchmark problem in question, there are no strong temperature variations. The continuity, momentum, and energy equations are:

$$
\begin{gathered}
\frac{\partial \rho}{\partial t}+\nabla \cdot(\rho \underline{u})=0 \\
\frac{\partial(\rho \underline{u})}{\partial t}+\nabla \cdot(\rho \underline{u} \underline{u})=-\nabla p+\mu \nabla^{2} \underline{u} \\
\frac{\partial(\rho E)}{\partial t}+\nabla \cdot(\rho H \underline{u})=\underline{u} \cdot \nabla p-\nabla \cdot(\underline{u} \cdot \underline{\tau})
\end{gathered}
$$

Closure relations for the energy $E$, and specific enthalpy $H$, are:

$$
\begin{gathered}
E=C_{v} T+\frac{|\underline{u}|^{2}}{2}, \\
H=C_{p} T+\frac{|\underline{u}|^{2}}{2}=E+\frac{p}{\rho} .
\end{gathered}
$$

\section{Numerical Model}

\section{A. Wind-US}

The present work adopts the NLES approach. The Wind-US code is employed herein to perform numerical Large-Eddy Simulation (LES) over a rectangular cylinder. As such, no SGS model is used, instead the numerical dissipation dampens the unresolved small scales. All the work herein pursues full three-dimensional physics. For the wake flow produced by flow over a sphere, the primary instability is two-dimensional in nature (Monkewitz 1987). Nevertheless, the downstream turbulent development evolves into a fully three-dimensional flow situation.

The simulations herein are performed using the Wind-US CFD code (Towne 2009). The spatial resolution of this study is fine enough such that most of the large scales of turbulence are captured directly. The numerical scheme used is the second-order upwinding Roe (1997) scheme for spatial discretization and the fourth-order Runge-Kutta scheme in time.

\section{B. Grid}

The simulations performed compare the fine mesh $(512 \times 512 \times 64)$ to the medium mesh $(256 \times 256 \times 32)$ in order to demonstrate grid independence. The total mesh size for the fine grid is 16 million points and for the medium grid is 2 million points, which is obtained by using every other point. The grid used is structured as seen from Figure 1 . The fine wall spacing near the cylinder is $\Delta y / D=0.0078$ which yields a cell Reynolds number of $24\left(R e_{c e l l}=\right.$ $\left.\rho U_{r e f} \Delta y / \mu\right)$. The inset of Figure 1 has a close-up of the corner demonstrating that $\Delta x=\Delta y$. Further away from the cylinder the grid is stretched. In a later section on the near wake region, Table 2 provides grid resolutions that correspond to the parametric study that varies streamwise depth and streamwise resolution.

\section{Boundary Conditions}

The operating parameters have been chosen to be the same as that of the experiments of Lyn et al. (1995). The Reynolds number is set at 22,000 to match the experiments $\left(R e_{D}=\rho U_{r e f} \mathrm{D} / \mu\right)$. The pressure difference between the inlet and outlet produces the farfield flow. The incoming stagnation pressure is $602.6494 \mathrm{kPa}$ while the downstream pressure at the exit is $602.2474 \mathrm{kPa}$. The free stream reference velocity is $U_{r e f}=9.31 \mathrm{~m} / \mathrm{s}$ and the diameter $D$ is taken to be $6.68 \mathrm{~mm}$ to yield the same Reynolds number as in the experiment. The Flow Through Time is defined as: $1 F T T=20.5 D / U_{\text {ref }}$, where the longitudinal length of the domain is $20.5 \mathrm{D}$ as evident in the bottom view of Figure 1. Note that the top view of Figure 1 illustrates the streamwise coupling at the boundaries. Its purpose is to simulate an infinite cylinder and remove any wall effects that would alternatively be present in an experimental wind tunnel. Although periodic boundary conditions are imposed in the spanwise direction, that alone cannot guarantee accurate capturing of the three-dimensional nature of vortex shedding. To avoid imposing a nonphysical spanwise mode, the spanwise extent and resolution must be adequate enough to capture spanwise wavelengths on the order of the shedding vortex. 


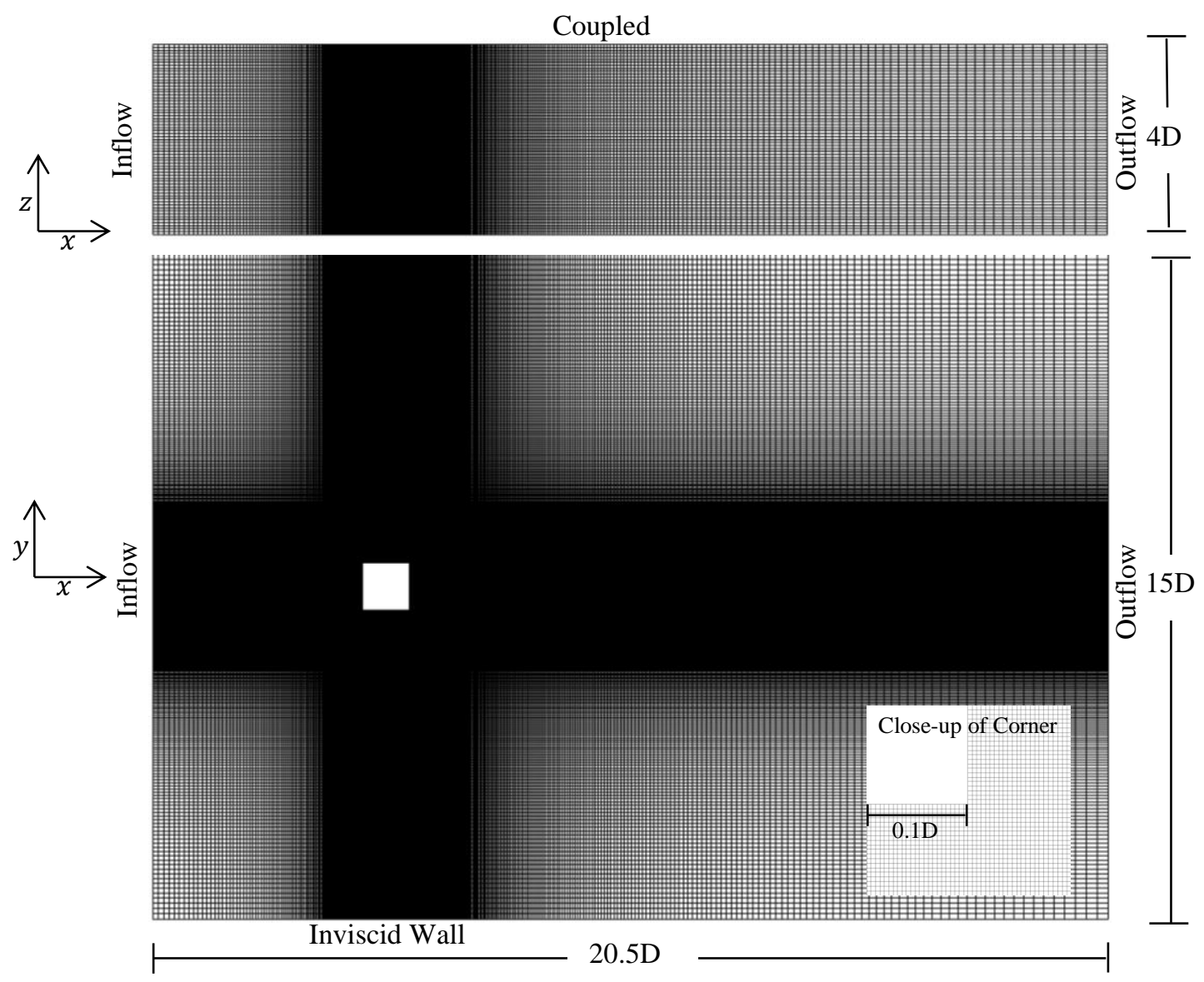

Figure 1: The boundary conditions and dimensions of the square cylinder for the fine grid.

\section{Results and Discussion}

The results are split into three sections: A) General description of the flow, B) Numerical sensitivities to mesh size, time step, and sampling frequency, and C) Parametric study of the effects of spanwise domain extent and spanwise grid spacing.

\section{A. General Description of Flow}

These computations were run on NASA's Pleiades supercomputer. The typical CPU runtime to obtain statistics for the medium mesh is about 3days. An isosurface of instantaneous vorticity modules is depicted in Figure 2. These instantaneous flow fields show the fully three dimensional nature of the wake flow. Figure 3 depicts the vortex shedding in the wake at different times. Note that there are large-scale structures that are on the order of the cylinder's diameter. Such structures need sufficient spanwise depth, truncating the domain too early would not sufficiently resolve the large z-wavelength behaviors. In Figure 4, instantaneous contours of streamwise velocity and the transverse velocity are shown. The size of the wake produced by the cylinder is evident. As expected, within the near wake region there are negative axial velocities. Note the transverse velocity is active as the flow turns over the corners and in the wake region. The aforementioned spanwise boundary conditions are also evident in the top view of the transverse velocity. 


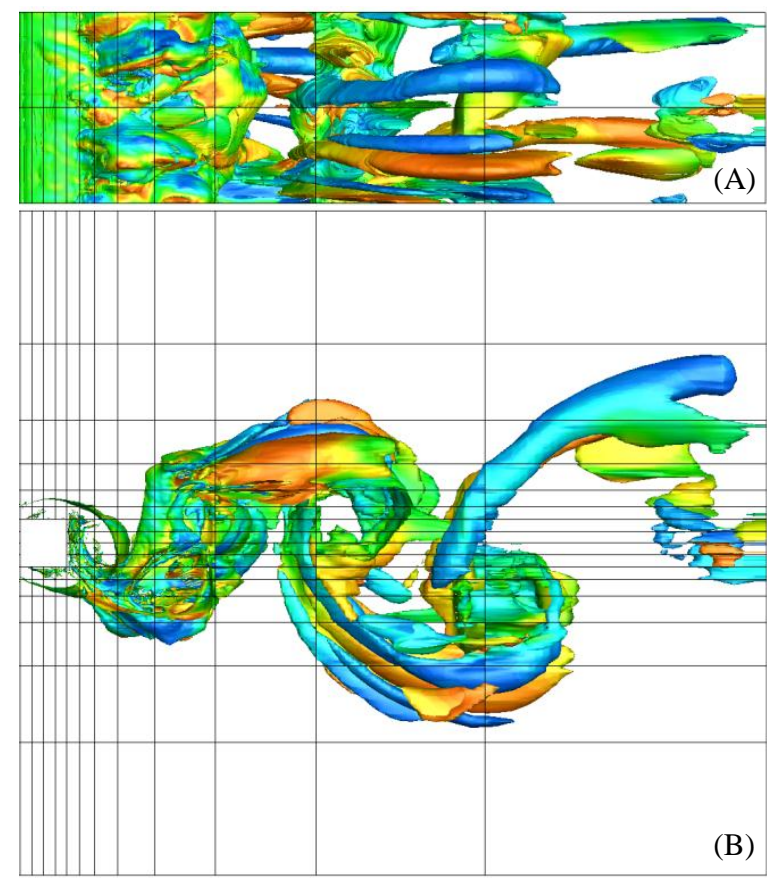

Figure 2: (Color online) Isosurface of instantaneous vorticity modules $\omega_{n}=1.1 U_{c} / D$ colored by the longitudinal vorticity $\omega_{x}$ in $\left.A\right)$ top and $B$ ) side views.

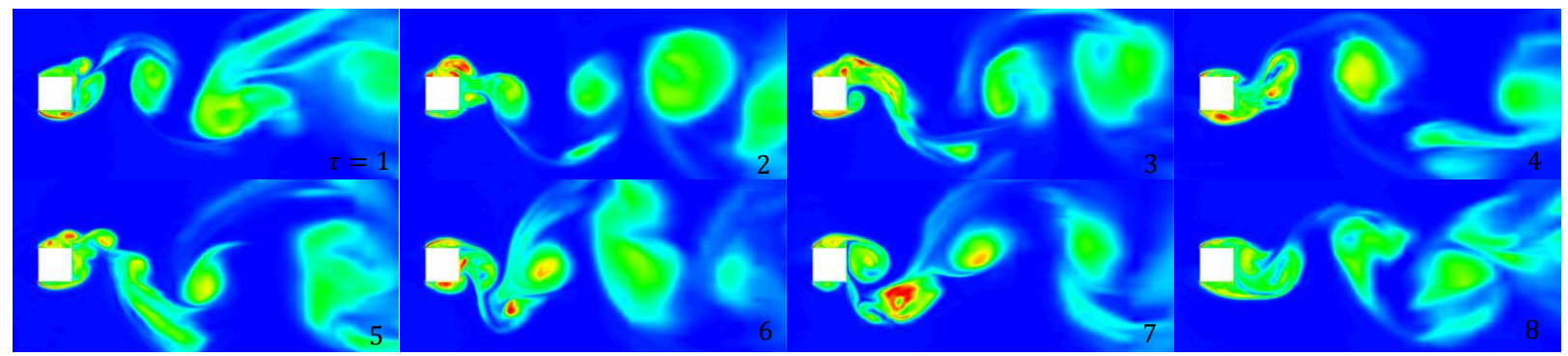

Figure 3: Entropy contours showing vortex shedding in the wake.
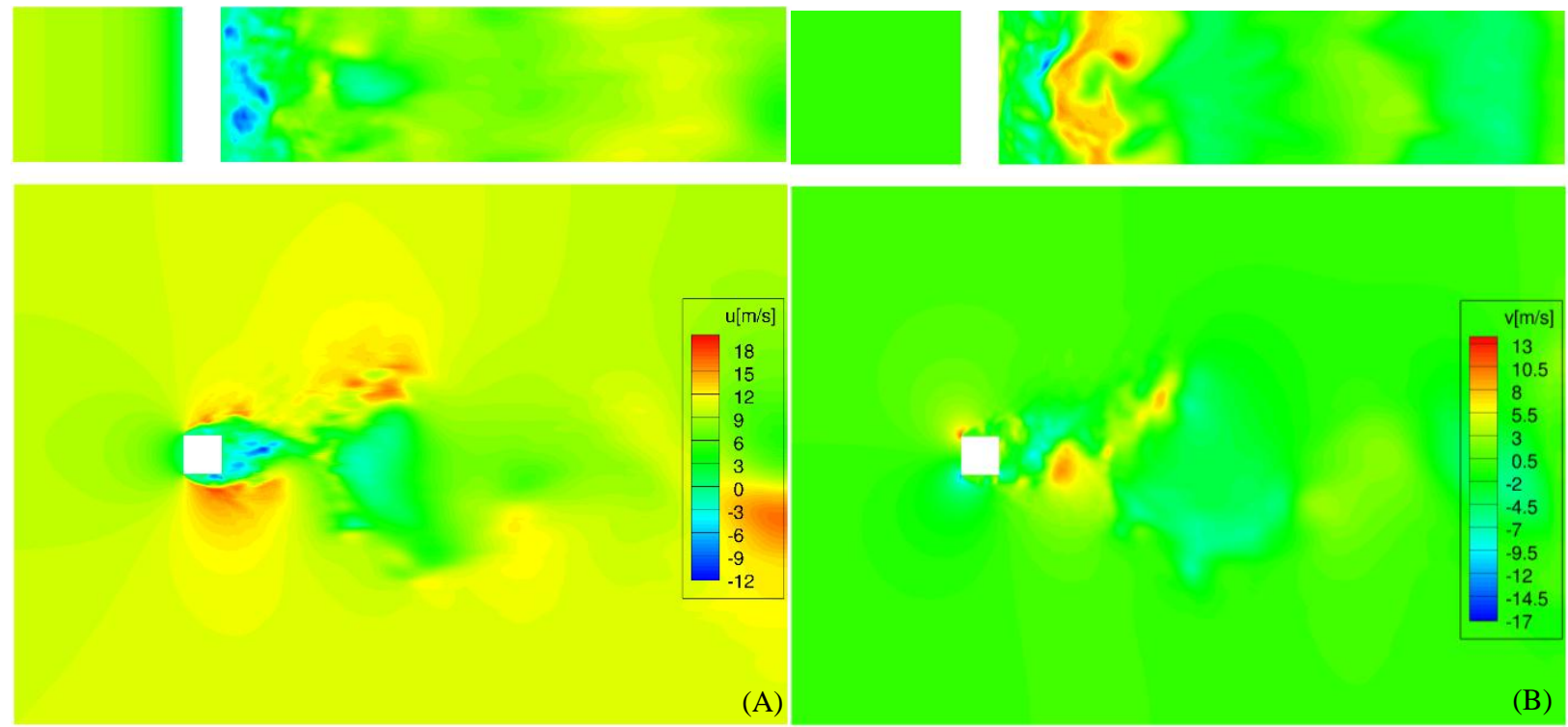

Figure 4: The instantaneous contour of A) the u-velocity and B) the v-velocity. 


\section{B. Numerical sensitivities to mesh size, time step, and sampling frequency}

The mesh size study was done at the Reynolds number of the 22,000 of the experiments. However, the timestep and sampling frequency studies were done at a lower Reynolds number of 3,000 (Senda et al. 1999). Despite this discrepancy the findings of these studies are still noteworthy.

\section{Mesh Size}

Table 1 lists characteristics of the baseline cases such as the spanwise width, sampling time, time step, and mesh size. A start-up time of 7FTT is sufficient enough to eliminate all transients. All of the following simulations use sampling times of atleast 5lFTT. Figure 5 is a comparison of both the 16 million point grid and the 4 million point grid with the experimental data. The data collected is along the flowfield centerline and the cylinder is seen between an $x / D$ of $[-0.5,0.5]$. Evidently the mean quantities of the two grids are close, with the exception of the near wake region. In that region, the peak negative velocity in the wake of the finer mesh is slightly higher since there are more points along its sharp gradient. Likewise the peak rms velocities of the two grids differ slightly, but match better further downstream. The overall recovery trend is the same for the experiment and simulations.

Table 1. Characteristics of baseline cases

\begin{tabular}{cccc}
\hline spanwise width & sampling time $(F T T)$ & $d t(\mu s)$ & Grid \\
\hline $5 \mathrm{D}$ & 51 & 1.0 & $256 \times 256 \times 64$ \\
$5 \mathrm{D}$ & 51 & 1.0 & $512 \times 512 \times 64$ \\
\hline
\end{tabular}
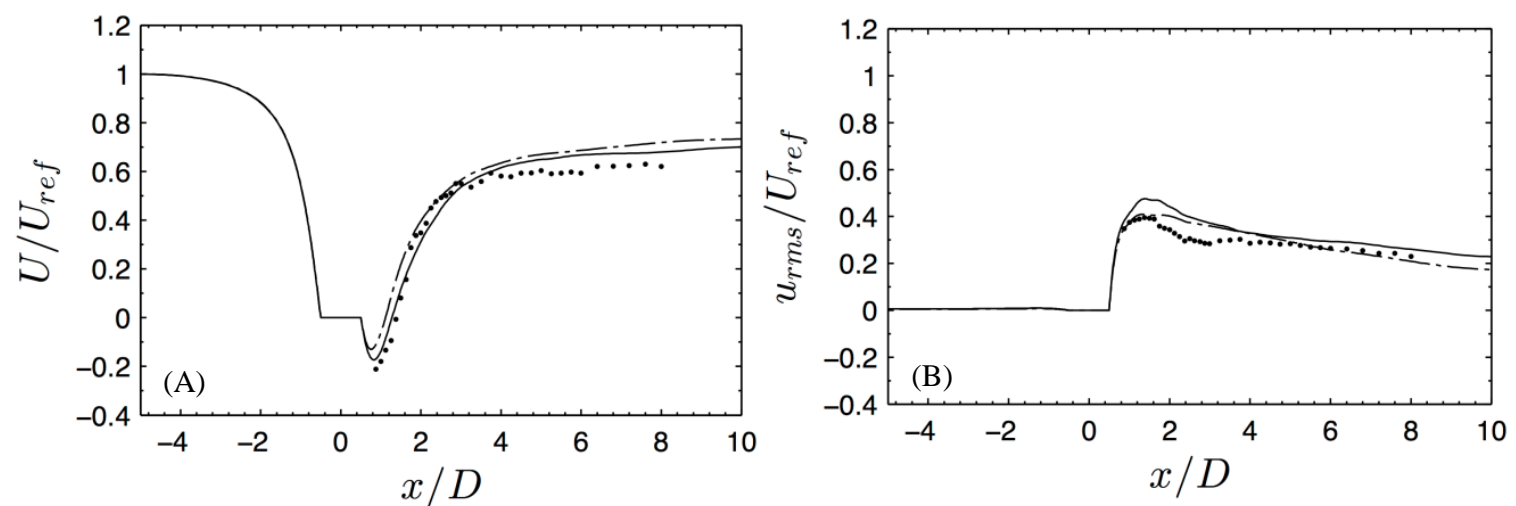

Figure 5: The effect of spatial resolution on the A) mean velocity and B) rms velocity on the flow field centerline. Experimental data.(circles); 512x512x64 NLES (solid line); 256x256x64 NLES(dashed dotted line).

Figure 6 shows the spectra of the transverse velocity fluctuations at four locations downstream of the square cylinder on the centerline of the wake. Evidently increasing mesh resolution has two effects: a) increased extent of well resolved frequencies and $b$ ) reduced the peak frequencies. This reduction might be due the smaller scales extracting energy from the larger scales. Additionally, this is the spectra of the transverse velocity fluctuations; when refining the mesh energy may be diverted to the spanwise fluctuations. The fundamental frequency, or vortex shedding frequency $f_{v s}$, is shown at $f / f_{v s}=1$. For $x / D=3$ and $x / D=5$, the odd harmonic of $f / f_{v s}=3$ is very pronounced. Computing the power spectra of fluctuations of the tangential velocity can also show the even harmonics at $f / f_{v s}=2,4$. The fundamental frequency is $160.6 \mathrm{~Hz}$ corresponding to the Strouhal number of 0.112 $(S t=f D / U)$. The experimental Strouhal number is 0.132 .

Adherence to the five-thirds power law is demonstrated at both levels. In Figure $6 \mathrm{~B}$, the better agreement with the $5 / 3$ law is due to better capturing the smaller scale eddies before they dissipate away into heat. The frequency spectra were collected over 51 FTT. The spectra were computed two ways: 1) with all the data collected over 51FTT and 2) with only half of the data over 25FTT. For method 2, only the very low frequency behaviors were slightly affected but nothing noticeable. Furthermore when the startup time was increased and the spectra were computed over only the last 25FTT, there was little or no change. These exercises were performed to confirm that the sampling time was sufficiently long enough and the startup time was not too soon. All the simulations herein have a minimum startup time of 6.8FTT and the statistics have been averaged over a minimum interval of 51FTT. Both of which are more than sufficient. Such large integration times have been selected to eliminate any numerical uncertainty that may arise from too short integration times and collected statistics too soon. 

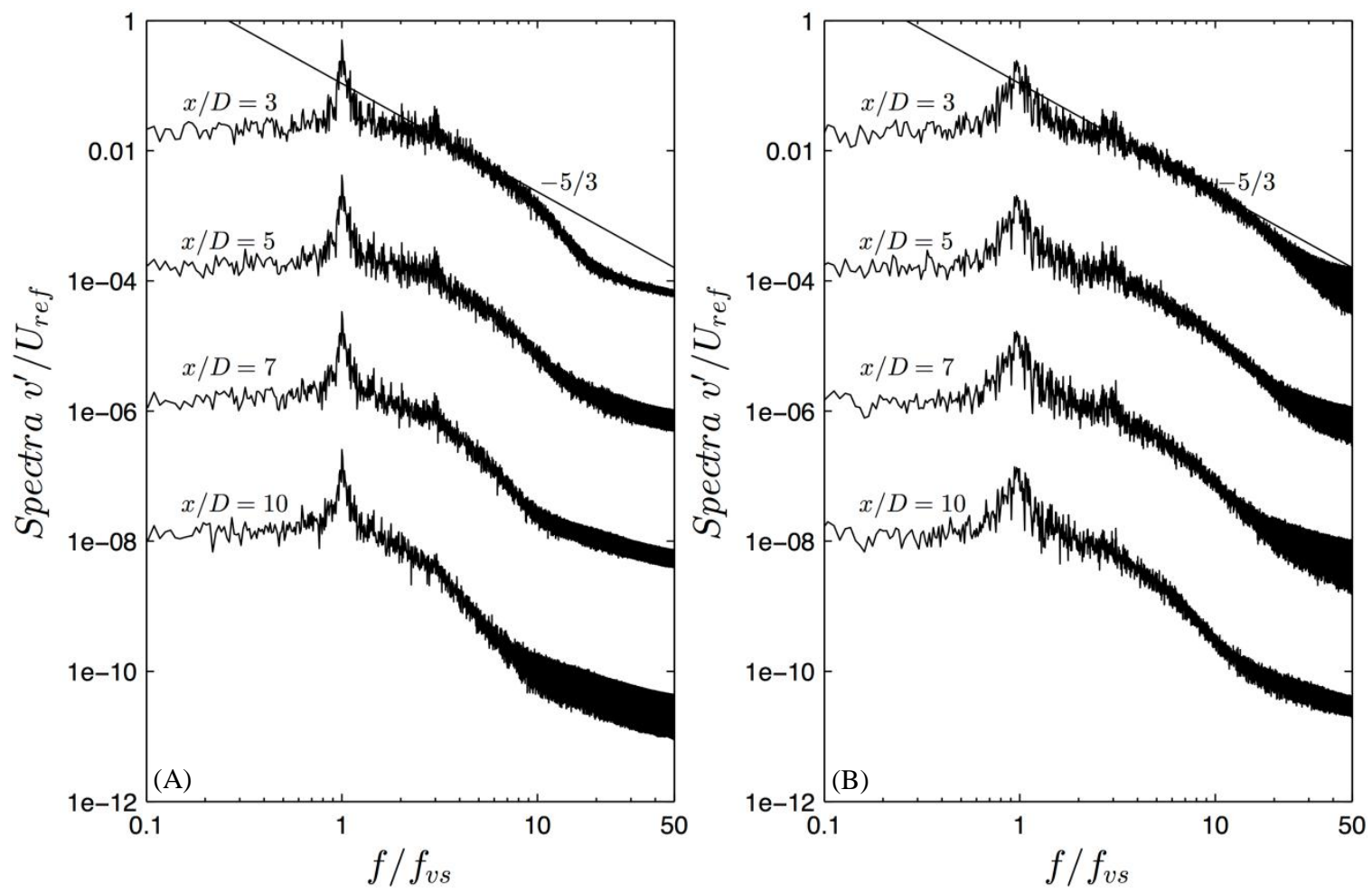

Figure 6: Spectra of the transverse velocity fluctuations at four locations on the flowfield centerline (the first at $x / D=3$ is not shifted) for A) the $256 \times 256 \times 64$ case and B) the $512 \times 512 \times 64$ case.

\section{Time Step}

The total calculation time is on the order of one second. A typical simulation enabled approximately 51 FTT, during which second-order statistics are collected. Figure 7 shows the effect of time-marching. The centerline mean velocity is shown in Figure 7, where it has been non-dimensionalized by the reference velocity $U_{\text {ref }}$. The flow is seen exiting the domain at $x / D=20.5$ with $U / U_{\text {ref }}=0.7$. Note that there are four lines in Figure 7 depicting the time step independence of the solution. The fourth-order Runge-Kutta (RK4) method is compared with the standard Euler step for two different values of $d t=0.5 \mu \mathrm{s}$ and $d t=1 \mu \mathrm{s}$. Note that for the smaller time step $d t=0.5 \mu \mathrm{s}$, the RK4 and Euler step are identical. Whereas for a larger time step of $1 \mu s$, the Euler and RK4 differ slightly. The effect of scheme is negligible so long as a small enough time step is taken. Note that the effect of $d t$ will change with grid size.

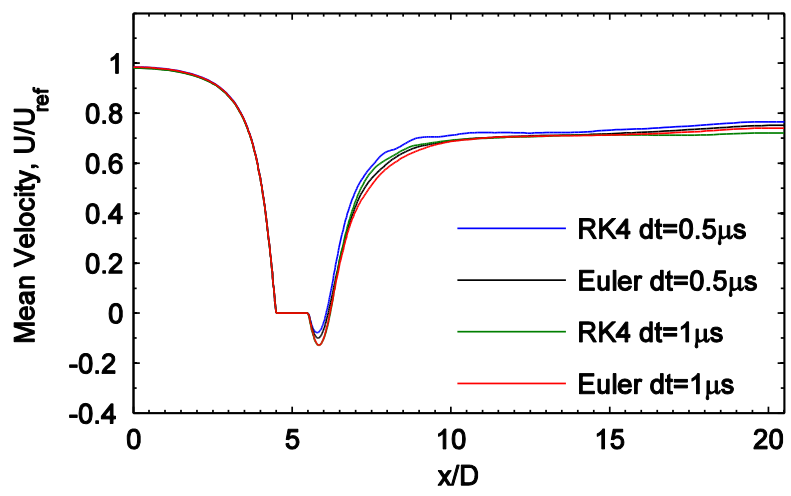

Figure 7: The time marching of streamwise mean velocity.

\section{Sampling}

Second-order statistics must be gathered at a sufficiently high enough frequency. The Nyquist criterion requires that the sampling frequency be twice the highest frequency captured. The shedding frequency is about $160 \mathrm{~Hz}$, 
consequently a sampling frequency of $50 \mathrm{kHz}$ contains 312 frames. In Figure 8, note the near-perfect match as the sampling frequency is increased from $12.5 \mathrm{kHz}$ to $100 \mathrm{kHz}$. Although there is no effect on the rms values as the sampling frequency increases, it affects the spectra's highest frequency which is half the sampling frequency.

Regarding the uncertainties produced by gathering statistics, it is well known that the duration for which statistics are gathered can add a small uncertainty regarding the second order statistics. In other works, the statistics were typically gathered over only 7 flow through times or over 10 shedding cycles. However in order to minimize such uncertainty, the statistics were collected over 51 FTT rather than seven. To reduce the statistical uncertainty, data was collected over large integration times thereby increasing the computational cost.

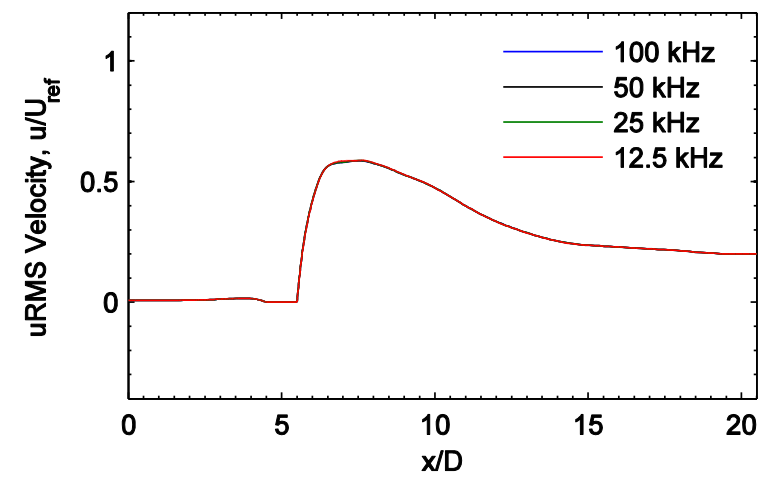

Figure 8: Sampling frequency invariance.

\section{Parametric study of the effects of spanwise domain extent and spanwise grid spacing}

After careful study of the numerical literature produced over the past decade, there have been several works that do not accurately capture the full 3D structures. Typically a $2 \mathrm{D}$ simulation is simply expanded into the third dimension without consideration of the spanwise direction and the isotropic conditions near the wall $(\Delta x=\Delta y=$ $\Delta z)$ are not commented on. It is well known that studies that meet the grid isotropic conditions tend to capture more of the real flow physics. Likewise, the fine resolution in 2D is typically wasted if it is not matched in the third dimension. Two factors can influence the accuracy of these results as much as any other: 1) the spanwise length relative to the largest vortices scale and 2) how well the grid isotropy condition is met. The effects of the spanwise domain extent have been well captured, however the spanwise grid resolution should be varied to gauge its significance.

\section{Effects of Spanwise Domain Extent}

\section{a)The Near Wake Region}

The z-depth of the previous section was fixed at 5D. The work herein will consider the effects of increasing the spanwise extent. To isolate the effects of grid spacing from domain extent it is necessary to fix $\Delta z$. Holding $\Delta z$ fixed at $5 \mathrm{D} / 64$, the effects of increasing the extent of the domain from 2.5D to 20D are shown in Figure 9. From Figure $9 \mathrm{~A}$ and 9E, it is evident that increasing the spanwise domain does not have a pronounced effect on the mean streamwise velocity and the TKE. However, a small domain of $2.5 \mathrm{D}$ causes an unnatural energy exchange between $u_{r m s}$ and $w_{r m s}$. The energy belonging to $w_{r m s}$ is deposited into $u_{r m s}$, thereby artificially increasing $u_{r m s}$ and decreasing $w_{r m s}$. This finding is in agreement with Rodi et al. (2006) observations that the original extent of $2 \mathrm{D}$ is insufficient.

Figure 9B depicts the excellent agreement between the 20D case and the experiments of Lyn et al. (1995). Notice how the blue 2.5D line is too high whereas the red 20D line has $u_{r m s}$ that closely follow the data. Increasing the spanwise extent has almost no effect on $v_{r m s}$ as evident in Figure 9C, the energy exchange is only between $u_{r m s}$ and $w_{r m s}$. From Figure 9D, it is evident that there is a noticeable difference between the 5D and 10D cases, but between 10D and 20D there is a less noticeable difference. Thus a 10D width is preferred for reasons of computational cost. 

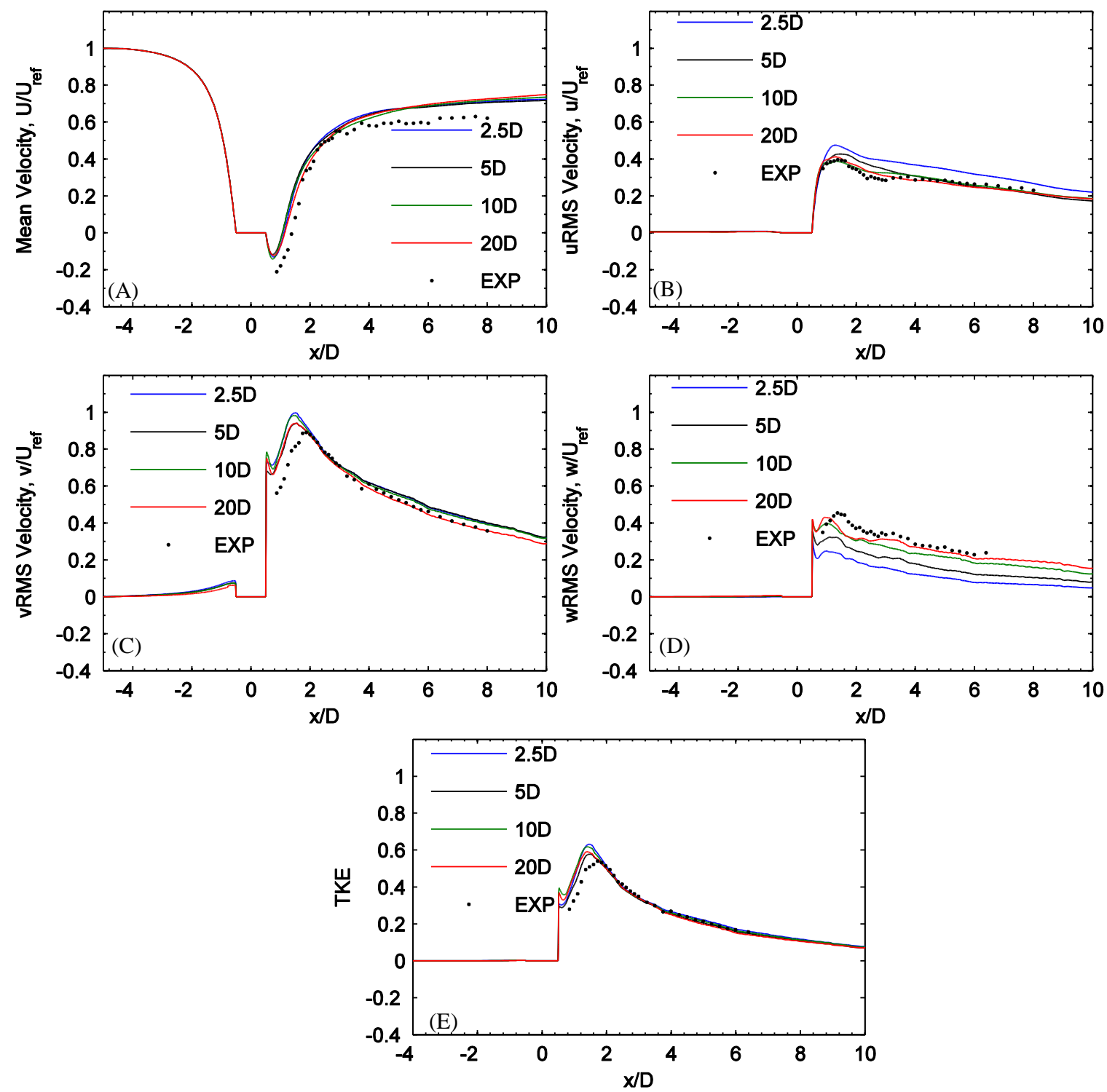

Figure 9: The effect of domain's extent in the spanwise direction on A) mean streamwise velocity $B) u_{r m s}$ velocity C) $v_{r m s}$ velocity $\left.D\right) w_{r m s}$ velocity and E) turbulent kinetic energy (TKE).

\section{b)The Near Wall Region}

In the near wall region, the flow is highly separated. Consequently there are large regions where reverse flow is evident. Figure 10 depicts the flow separation. The boundary layer profile is sampled at four different stations $x / D=\left[\begin{array}{lll}-0.25 & 0.0 & 0.250 .5\end{array}\right]$. Figure 11 compares the mean velocity profile with that of the experiments of Lyn et al. (1995). They match fairly well with experimental data. The experimental mean velocity data tends to be slightly less than that of the simulations closer to $x / D=-0.25$. This observation was also seen by that of Matthieu at al. (2008). Figure 12 compares streamwise stress profiles to the experiment. Note that as you move downstream of the leading edge (increasing $x / D$ ), the rms streamwise stress increases as the separation intensifies. The rms values of the streamwise stress tend to agree very well with experimental data, however there is a large value of rms in the near wall region that the experimental measurements may not have identified. The first peak in rms quantities corresponds to the location of the maximum shear stress in the reversed flow part of the boundary layer, which corresponds to an inflection point in the mean velocity between 0 and -0.5 . Note there is another inflection point in mean velocity as it goes from -0.5 to almost 1.5 (Figure 11C), which corresponds to the second peak seen in the rms values furthest away from the wall (Figure 12C). The original LES of Rodi et al. (1997) also predicted a large rms value in the near wall region. 
As expected, the spanwise parametric study has a more pronounced effect on the near wake region rather than the near wall region. Although expanding the depth of the cylinder does not have a significant effect on the mean profiles, its effect is better seen in the streamwise stress term $\overline{u^{\prime} u^{\prime}}$. As the depth is increased better agreement is reached at $y / D \sim 1$. Also the local peak $y / D \sim 0.6$ is significantly reduced as the depth is gradually increased from 2.5D to 20D. The experimental data of Lyn et al. (1995) did not measure any $w_{r m s}$ values and only provided 2D TKE measurements. Such data might help reduce uncertainties especially at the flow corners.

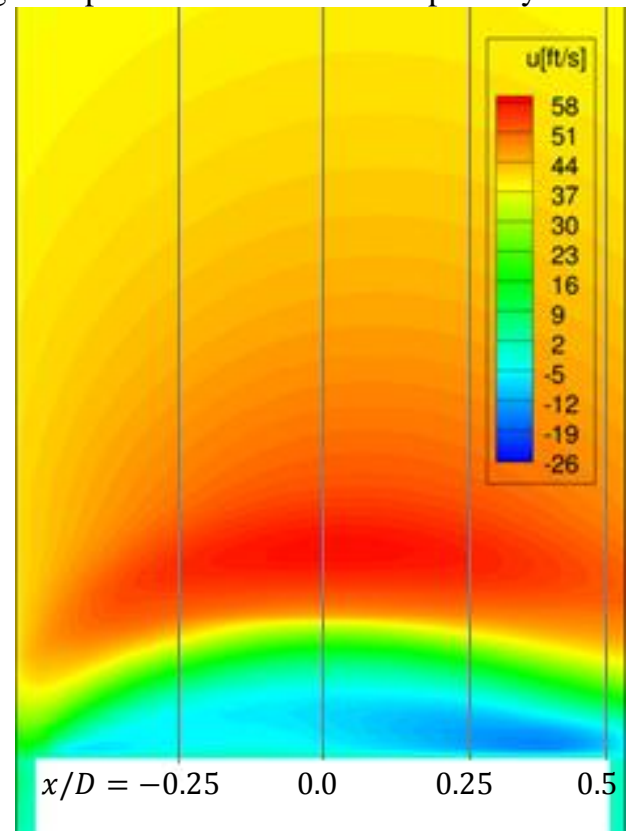

Figure 10: Flow separation in the near wall region on the topside.
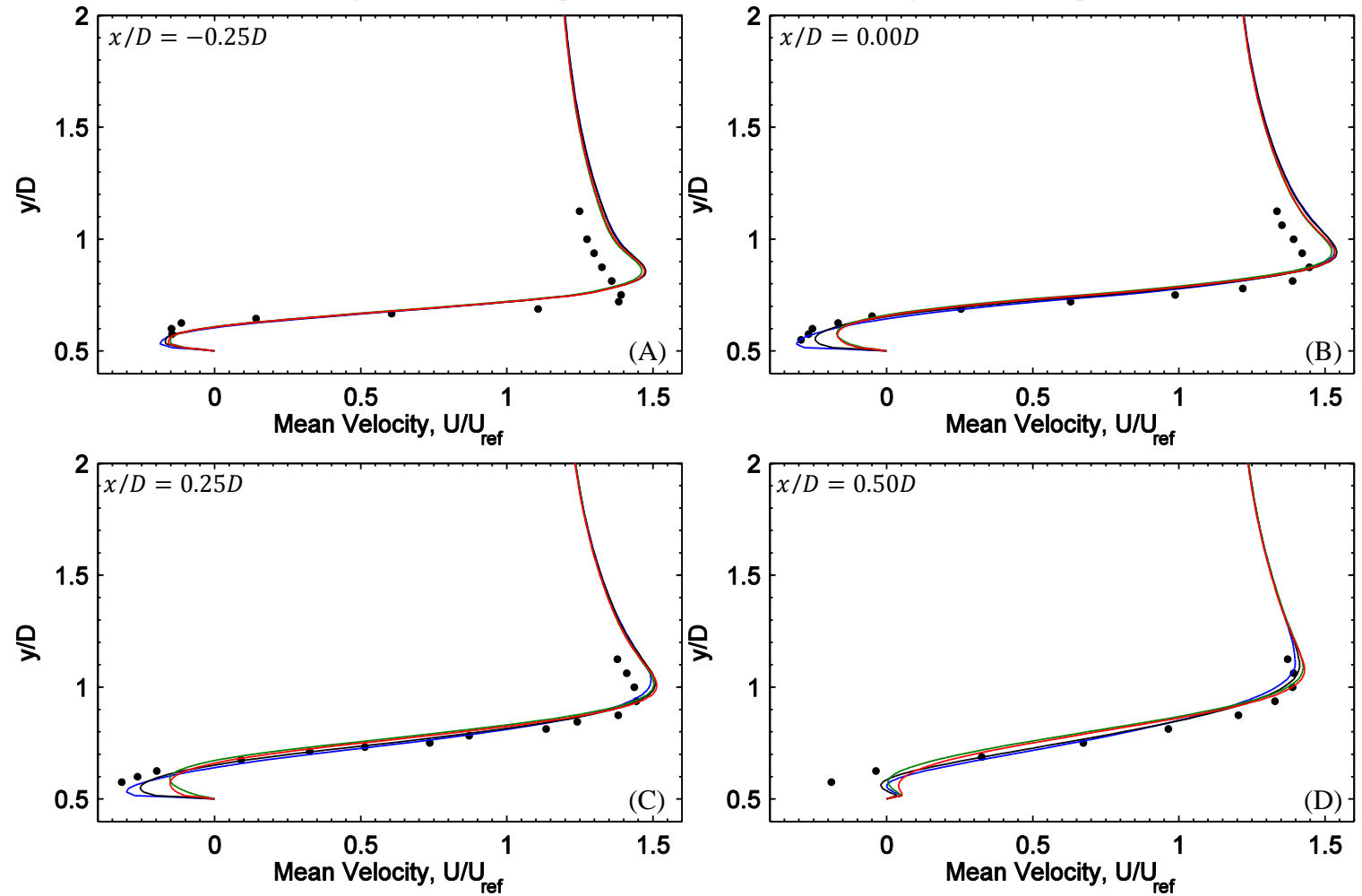

Figure 11: The mean-velocity in the near wall region at A) $x / D=-0.25 D$ B) $x / D=0.00 D C$ ) $x / D=0.25 D$ and $D$ ) $x / D=0.50 D$. (Legend: EXP circular dots, spanwise width of 2.5D [blue], 5D [black], 10D [green], 20D [red].) 

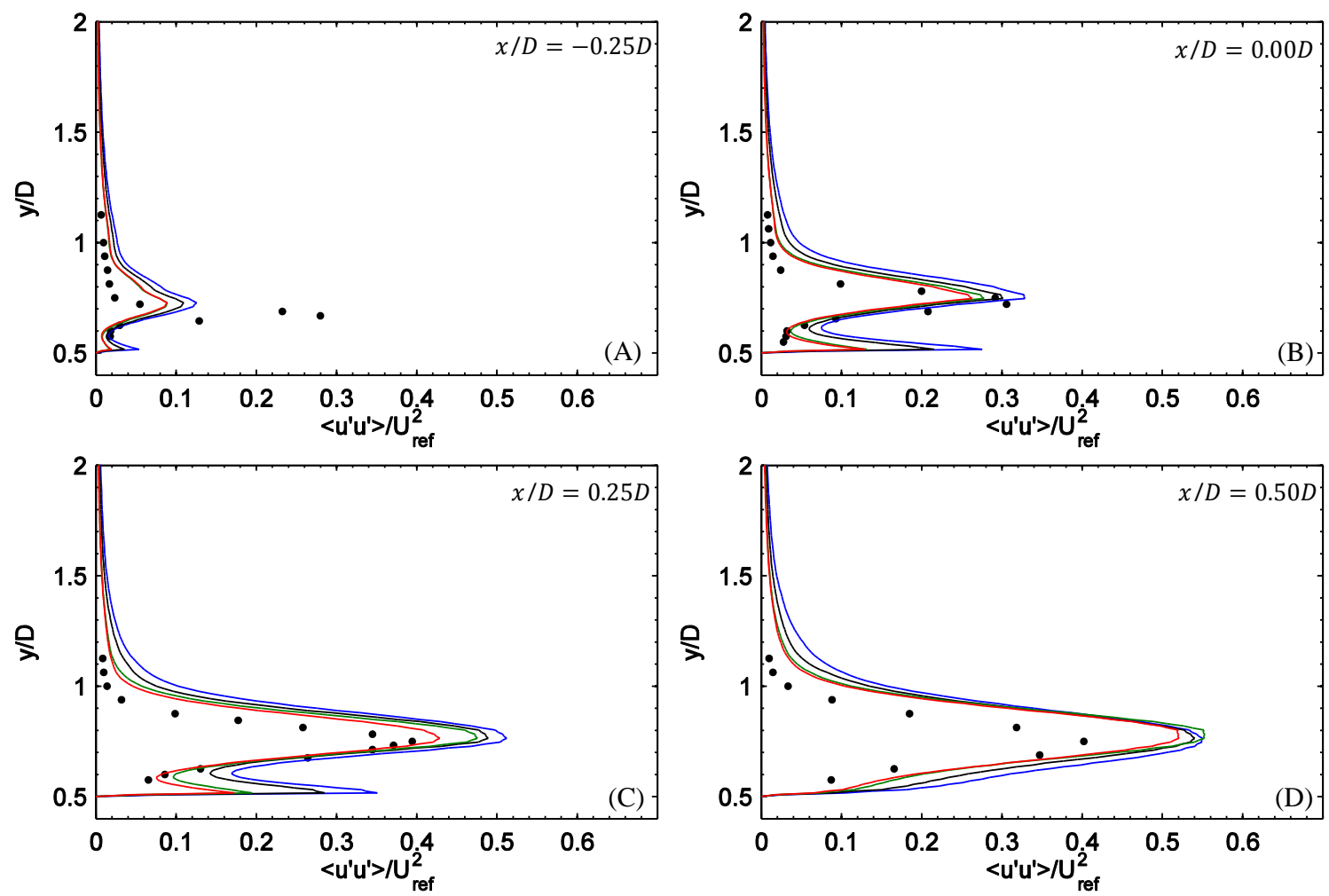

Figure 12: The streamwise stress term $\overline{u^{\prime} u^{\prime}}$ in the near wall region at A) $x / D=-0.25 D B$ ) $x / D=0.00 D$ $C$ ) $x / D=0.25 D$ and $D$ ) $x / D=0.50 D$. (Legend: EXP circular dots, spanwise width of $2.5 D$ [blue], $5 D$ [black], $10 D$ [green], 20D [red].)

Table 2 lists global aerodynamic parameters such as Strouhal number, drag coefficient, rms drag coefficient, rms lift coefficient, reattachment length, and the grid size. The Strouhal number of shedding is computed based on the periodic lift of the cylinder. The fast Fourier transform of the instantaneous lift coefficient across many FTTs yields a peak frequency of $160.6 \mathrm{~Hz}$ corresponding to the Strouhal number $S t=f D / U$. From Table 2, the computed $S t$ for the present LES 20D case is 0.112 which is compared to that of the experiments of 0.132 (see Rodi 1997). The coefficient of drag is 2.2686 which is comprable to that of Lyn's experiments 1.9-2.2. Note that $C_{d_{r m s}}$ and $l_{R}$ have large disagreement with experiment at $2.5 \mathrm{D}$ and improve with wider spans.

Table 2. Global aerodynamic parameters

\begin{tabular}{|c|c|c|c|c|c|c|c|}
\hline \multicolumn{2}{|c|}{ Solution Method } & St & $\bar{C}_{d}$ & $C_{d_{r m s}}$ & $C_{l_{r m s}}$ & $l_{R}$ & Grid \\
\hline \multirow{2}{*}{\multicolumn{2}{|c|}{$\begin{array}{l}\text { Experiments } \\
\text { LES Rodi UK2 }\end{array}$}} & 0.132 & $1.9-2.2$ & $0.1-0.2$ & & 1.38 & \\
\hline & & 0.13 & 2.3 & 0.14 & 1.15 & 1.4 & $146 \times 146 \times 20$ \\
\hline \multirow{4}{*}{ Present LES } & $2.5 \mathrm{D}$ & 0.121 & 2.1716 & 0.348 & 1.25 & 1.093 & $256 \times 256 \times 32$ \\
\hline & 5D & 0.115 & 2.1627 & 0.259 & 1.14 & 1.1 & $256 \times 256 \times 64$ \\
\hline & $10 \mathrm{D}$ & 0.109 & 2.2789 & 0.242 & 1.08 & 1.13 & $256 \times 256 \times 128$ \\
\hline & $20 \mathrm{D}$ & 0.112 & 2.2686 & 0.154 & 0.84 & 1.14 & $256 \times 256 \times 256$ \\
\hline
\end{tabular}

\section{Effect of Spanwise Grid Spacing}

In the aforementioned comparison, $\Delta z$ was fixed at $5 \mathrm{D} / 64$ and the extent of the domain was varied. This section discusses studies where the extent of the spanwise domain was fixed at 5D and $\Delta z$ was varied. This way, the effect of meeting the isotropic grid resolution criteria in the near wall region is isolated from the domain's extent effects. From Figure 13, it is evident that the higher resolution matches the experimental data better.

Examining the results plotted in Figure 13, essentially when only 16 or 32 points are used in the spanwise direction, vortices in the third dimension are not being resolved. The lack of the resolution artificially raises $u_{r m s}$ and dampens $w_{r m s}$. In such a scenario the simulations are indeed $3 \mathrm{D}$, however due to the limitations, they are only capturing 2D physics. The 64points case however has good grid isotropy with a $\Delta z / D=0.0781$ and the wall 
spacing of the medium mesh is $\Delta y / D=0.016$. A further improvement in grid isotropy for 128 points $(\Delta z / D=$ 0.0391 ) could yield further improvement. Based on Hybrid RANS-LES simulations for a turbulent supersonic shear layer, by Georgiadis et al. (2003), the total spanwise width of 1-2 mm was found to prohibit the formation of any large-scale turbulence. When the domain was extended to $4-6 \mathrm{~mm}$, the growth of spanwise-varying turbulence was found to begin, but that study concluded a much wider spanwise domain was required. The observations herein agree in that both the extent of the domain and the spanwise grid resolution $(\Delta z)$ are key parameters that affect the accuracy of LES simulations.
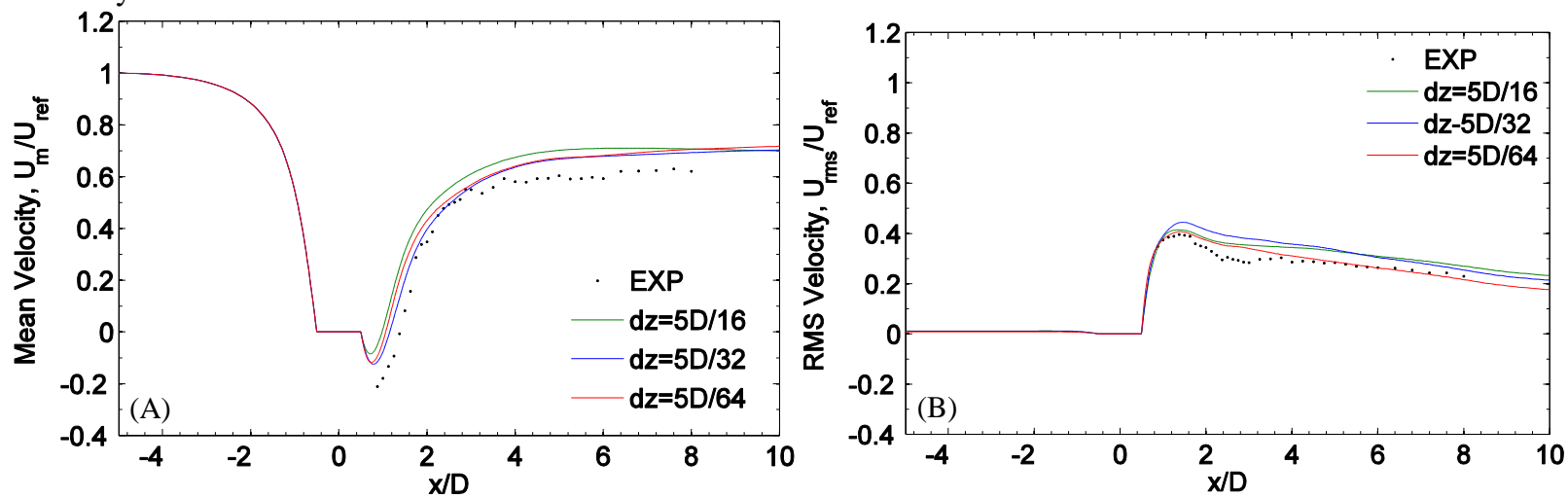

Figure 13: The effect of spanwise grid resolution on A) mean streamwise velocity and B) u-rms velocity.

\section{Conclusions}

The flow over a square cylinder was simulated using the NLES approach. This work examined the numerical sensitivities to mesh size, time step, and sampling frequency. Next, a parametric study was performed to examine the effects of the spanwise domain extent and grid spacing on the near wake and the near wall regions. Increasing the mesh resolution resulted in a better agreement with the $5 / 3$ power law as the smaller scales were better resolved. The comparison of the streamwise mean and rms profiles of the two million, sixteen million, and experiments showed good agreement. Once a sufficiently small enough time step is chosen, whether it is RK4 or an Euler step had little effect. As the sampling frequency was increased from $12.5 \mathrm{kHz}$ to $100 \mathrm{Khz}$ there was no change in the rms profile.

The spanwise width was then varied from the original 5D. Keeping the spanwise spacing fixed, four cases were compared with span widths of $[2.5,5,10,20] \mathrm{D}$. As the width was increased from 5D to 20D, higher spanwise modes were being resolved, consequently, the $w_{r m s}$ increased while $u_{r m s}$ decreased. This energy exchange is expected since large-scale structures dominate the flow, a short spanwise width of $2.5 \mathrm{D}$ constricts large eddies from forming. There was a noticeable difference in $w_{r m s}$ between the 5D and 10D cases but a less significant change between the 10D and 20D cases. Therefore the 10D spanwise width is preferred and it is affordable thanks to the increase in computational power. For higher Reynolds numbers, the dominant shedding length-scale becomes smaller and thus a narrower domain may suffice.

After characterizing the near wake region, attention was turned to the near wall region where the flow is separated. The peak rms values compared favorably well with the experimental data. Expanding the domain in the $\mathrm{z}$ direction, allows for minute small $w_{r m s}$ values to occur within the separation region, this in turn explains why the $20 \mathrm{D}$ case had a smaller peak streamwise-stress value than that of the $2.5 \mathrm{D}$ case. Extending the domain had no effect on the base profiles of the separated boundary layer.

In the aforementioned comparisons, $\Delta z$ was fixed at $5 \mathrm{D} / 64$ in order to capture the high frequency modes in the near wake region. A coarser resolution would not be able to fully capture the high frequency unsteady behavior in the spanwise direction. Refining in $\Delta z$ while keeping the width fixed, helped distinguish the effects of better grid spacing in the near wall region from the domain's extent effects.

The effect of spanwise width is rarely considered in LES of flows that are two-dimensional in the mean sense. Although the mean flow can in some cases be accurately captured with insufficient spanwise length and resolution, this work shows that the turbulence statistics require a sufficiently wide domain extent and spanwise resolution. As demonstrated herein, an insufficient spanwise extent results in over prediction of the streamwise and transverse turbulent intensities and correspondingly artificial suppression of the spanwise turbulent intensity. The NLES predictions matched the experimental data. With the increase in computational performance, future LES studies will hopefully properly address the significance of the spanwise extent as turbulent wake flow is highly three dimensional in nature. 


\section{Acknowledgments}

This work was supported by the Rotary Wing project and the Aeronautical Sciences project under the Fundamental Aeronautics Program. Special thanks to J. R. DeBonis, D. A. Yoder, and G. E. Welch for their advice.

\section{References}

[1] S. Nakagawa, K. Nitta, M. Senda, "An experimental study on unsteady turbulent near wake of a rectangular cylinder in channel flow," J. Exp. Fluids, 27 (3) (1999), pp. 284-294.

[2] J. R. DeBonis, "Progress Toward Large-Eddy Simulations for Prediction of Realistic Nozzle Systems," J. Propulsion and Power, Vol. 23, No. 5, Sept. 2007, pp. 971-980.

[3] J-P. Chollet, P. R. Voke, and L. Kleiser, "Direct and Large-Eddy Simulation II," Proceedings of the ERCOFTAC Workshop held in Grenoble, France, 16-19 September 1996.

[4] P. A. Monkewitz, and L. N. Nguyen, "Absolute Instability in the Near-Wake of Two-Dimensional Bluff Bodies," J. Fluids and Structure, Vol. 1, pp. 165-184, 1987.

[5] C. E. Towne, "Wind-US User's Guide, Version 2.0," NASA/TM-2009-215804, October 2009.

[6] D. A. Lyn, S. Einav, W. Rodi, and J. H. Park, "A laser-Doppler velocimetry study of Ensemble-averaged characteristics of the turbulent wake of a square cylinder," J. Fluid Mech., 304, 285 - 319, 1995.

[7] W. Rodi, "Comparison of LES and RANS calculations of the flow areound bluff bodies," J. Wind Engineering, 69.71, 55 $-75,1997$.

[8] N. J. Georgiadis, J. I. D. Alexander, and E. Reshotko, "Hybrid Reynolds-Averaged Navier-Stokes/Large-Eddy Simulations of Supersonic Turbulent Mixing," AIAA Journal, Vol. 41, No. 2, 2003, pp. 218-229.

[9] S. J. Eastwood, P. G. Tucker, H. Xia, C. Klostermeier, "Developing large eddy simulation for turbomachinery applications," Phil. Trans. R. Soc. A 367, (2009).

[10] C. Hah, H. W. Shin, "Study of Near-Stall Flow Behavior in a Modern Transonic Fan With Compound Sweep," J. Fluids Engineering, Vol. 134 / 071101 (2012).

[11] V. N. Rao - R. J. Loveday P. G. Tucker - S. Lardeau, "Large Eddy Simulations in Turbines: Influence of Roughness and Free-Stream Turbulence," Flow, Turbulence and Combustion 92:543-561, 2014.

[12] P.G. Tucker, "Computation of unsteady turbomachinery flows: Part 1-Progress and challenges," Elsevier: Progress in Aerospace Sciences, Volume 47, Issue 7, Pages 522-545, October 2011.

[13] P.G. Tucker, "Computation of unsteady turbomachinery flows: Part 2-LES and hybrids," Elsevier: Progress in Aerospace Sciences, Volume 47, Issue 7, Pages 546-569, October 2011.

[14] A. Gross, H. F. Fasel, "Strategies for Simulating Flow Through Low-Pressure Turbine Cascade," Journal of Fluids Engineering, Vol. 130 , Nov. 2008.

[15] D. P. Rizzetta, M. R. Visbal, "Plasma-Based Flow-Control Strategies for Transitional Highly Loaded Low-Pressure Turbines," ASME: Journal of Fluids Engineering, Vol. 130 / 041104-1, April 2008.

[16] D. P. Rizzetta, M. R. Visbal, "Numerical Investigation of Plasma-Based Flow Control for Transitional Highly Loaded LowPressure Turbine," AIAA Journal, Vol. 45, No. 10, October 2007.

[17] K. Matsuura, C. Kato, "Large-Eddy Simulation of Compressible Transitional Flows in a Low-Pressure Turbine Cascade," AIAA Journal, Vol. 45, No. 2, February 2007.

[18] J. Huang, T. C. Corke, F. O. Thomas, "Plasma Actuators for Separation Control of Low-Pressure Turbine Blades," AIAA Journal, Vol. 44, No. 1, January 2006.

[19] J. Huang, T. C. Corke, F. O. Thomas, "Unsteady Plasma Actuators for Separation Control of Low-Pressure Turbine Blades," AIAA Journal, Vol. 44, No. 7, July 2006.

[20] S. Sarkar, P. R. Voke, "Large-Eddy Simulation of Unsteady Surface Pressure Over a Low-Pressure Turbine Blade due to Interactions of Passing Wakes and Inflexional Boundary Layer," Journal of Fluids Engineering, Vol. 128 / 221, April 2006.

[21] W. Rodi, "DNS and LES of some engineering flows," Elsevier: Fluid Dynamics Research, 38, 145-173, 2006.

[22] J. G. Wissink and W. Rodi, "LES of Passive Heat Transfer in a Turbine Cascade," Institute for Hydromechanics, University of Karlsruhe, Kaiserstrasse 12, 76128 Karlsruhe, Germany, 2004.

[23] V. Michelassi, J. G. Wissink, J. Fr"ohlich, and W. Rodi, "Large-Eddy Simulation of Flow Around Low-Pressure Turbine Blade with Incoming Wakes," AIAA Journal, Vol. 41, No. 11, November 2003.

[24] B. Raverdy, I. Mary, P. Sagaut, N. Liamis, "High-Resolution Large-Eddy Simulation of Flow Around Low-Pressure Turbine Blade," AIAA Journal, Vol. 41, No. 3, March 2003.

[25] J. P. Boris, F. F. Grinstein, E. S. Oran, and R. L. Kolbe, "New insights into large eddy simulation," Fluid Dynamics Research, 10 (1992), 199-229. 